\title{
MULTIVALUED NONEXPANSIVE MAPPINGS AND OPIAL'S CONDITION
}

\author{
E. LAMI DOZO ${ }^{1}$
}

AbSTRACT. We give relations between a condition introduced by $Z$. Opial which characterizes weak limits by means of the norm in some Banach spaces and approximations of the identity, in particular for systems of projections. Finally a fixed point theorem for multivalued nonexpansive mappings in a Banach space satisfying this condition is proved; this result generalizes those of J. Markin and F. Browder.

1. Introduction. Let $X$ be a Banach space, $C$ a convex weakly compact subset of $X$ and $T$ a nonexpansive mapping from $C$ into $C$, i.e.

$$
\|T x-T y\| \leqq\|x-y\|, \quad x, y \text { in } C .
$$

Related to the problem of existence of a fixed point for $T$ and its approximation, Z. Opial [9] introduced an inequality for weak convergent sequences characterizing its limits; we take this property as

Definition 1.1. Let $X$ be a Banach space. $X$ satisfies Opial's condition if for each $x$ in $X$ and each sequence $\left\{x_{n}\right\}$ weakly convergent to $x$

$$
\lim \inf \left\|x_{n}-y\right\|>\lim \inf \left\|x_{n}-x\right\|
$$

holds for $y \neq x$.

An equivalent definition [2] is obtained replacing (1.1) by

$$
\lim \sup \left\|x_{n}-y\right\|>\lim \sup \left\|x_{n}-x\right\|,
$$

this following from

$$
\begin{aligned}
\lim \inf \left\|x_{n}-y\right\| & =\lim _{k}\left\|x_{n_{k}}-y\right\| \\
& >\lim \sup \left\|x_{n_{k}}-x\right\| \geqq \lim \inf \left\|x_{n}-x\right\|
\end{aligned}
$$

and from

$$
\begin{aligned}
\lim \sup \left\|x_{n}-x\right\| & =\lim _{j}\left\|x_{n_{j}}-x\right\| \\
& <\lim \inf \left\|x_{n_{j}}-y\right\| \leqq \lim \sup \left\|x_{n}-y\right\|
\end{aligned}
$$

for convenient subsequences of $\left\{x_{n}\right\}$.

Received by the editors November 30, 1971 and, in revised form, April 22, 1972. AMS (MOS) subject classifications (1970). Primary 47H10, 46B99; Secondary 54C60.

Key words and phrases. Fixed point theorem, systems of projections, geometric properties of Banach spaces.

1 These results are part of the author's doctoral thesis (University of Brussels, 1970).

(c) American Mathematical Society 1973 
Hilbert spaces and $l^{p}$ spaces $(1<p<\infty)$ satisfy Opial's condition, also finite dimensional Banach spaces.

The existence of a fixed point in the problem mentioned in the beginning of this paragraph is known when $X$ satisfies Opial's condition, but from results of [5] this is a corollary of a theorem of W. Kirk [6], so this condition becomes interesting in the approximation of a fixed point for a "univalued" nonexpansive mapping (see [9]).

A useful simplification of (1.1) is given in the following

Lemma 1.1. A Banach space $X$ satisfies Opial's condition if and only if

$$
x_{n} \rightarrow 0 \text { and } \lim \inf \left\|x_{n}\right\|=1 \Rightarrow \lim \inf \left\|x_{n}-x\right\|>1
$$

for $x \neq 0$, where $\rightarrow$ denotes weak convergence of the sequence $\left\{x_{n}\right\}$.

Proof. Let $y_{n} \rightarrow y$; if lim inf $\left\|y_{n}-y\right\|=0$, then (1.1) follows from the unicity of a weak limit. If not take $a=\lim \inf \left\|y_{n}-y\right\|, a>0$, and $x_{n}=$ $a^{-1}\left(y_{n}-y\right)$; then $x_{n} \rightarrow 0$ and $\lim \inf \left\|x_{n}\right\|=1$, so (1.3) gives

$$
\lim \inf \left\|x_{n}-x\right\|>1
$$

for $x \neq 0$. Replacing $x_{n}$ we obtain

$$
\lim \inf \left\|y_{n}-y-a x\right\|>\lim \inf \left\|y_{n}-y\right\|
$$

for $x \neq 0$, i.e. for any $y-a x \neq y$. The inverse implication is obvious. Q.E.D.

2. Approximation of the identity. We consider a directed family of continuous linear operators of finite rank which approach the identity of a Banach space $X$, i.e. a family $\left\{P_{j} ; j \in J, P_{j}\right.$ linear and continuous $\}$, where $J$ has an "increasing" order denoted by $\infty 0$ such that

$$
P_{j}: X \rightarrow X_{j}, \quad \operatorname{dim} X_{j}<\infty, \quad X_{j} \text { subspace of } X,
$$

$$
\lim P_{j} x=x, \text { for each } x \text { in } X .
$$

We shall call such a family an approximation of the identity. For example when a Banach space posseses a Schauder basis, the associated system of projections constitutes an approximation of the identity.

THEOREM 2.1. Let $X$ be a Banach space and let $\left\{P_{j} ; j \in J\right\}$ be an approximation of the identity. If for each $c>0$ there exists $r=r(c)>0$ such that

$$
\left\|x-P_{j} x\right\|=1 \text { and }\left\|P_{j} x\right\| \geqq c \Rightarrow\|x\| \geqq 1+r
$$

holds for $j \supset j_{0}, j_{0}$ fixed in $J$, then $X$ satisfies Opial's condition.

Proof. We apply Lemma 1.1 and verify (1.3), so we take $x_{n} \rightarrow 0$ with $\lim \inf \left\|x_{n}\right\|=1$ and $x \neq 0$. If we define $Q_{j}=I-P_{j}$, where $I$ is the identity on 
$X$, we have

$\left\|x_{n}\right\|-\left(\left\|P_{j} x_{n}\right\|+\left\|Q_{j} x\right\|\right) \leqq\left\|Q_{j}\left(x_{n}-x\right)\right\| \leqq\left\|x_{n}-x\right\|+\left\|P_{j}\left(x_{n}-x\right)\right\|$ and

Then

$$
\left\|P_{j} x\right\| \leqq\left\|P_{j}\left(x_{n}-x\right)\right\|+\left\|P_{j} x_{n}\right\|
$$

$$
\begin{aligned}
1-\left\|Q_{j} x\right\| & \leqq \liminf _{n}\left\|Q_{j}\left(x_{n}-x\right)\right\| \leqq \limsup _{n}\left\|Q_{j}\left(x_{n}-x\right)\right\| \\
& \leqq \lim _{n} \sup \left\|x_{n}-x\right\|+\left\|P_{j} x\right\|, \\
\left\|P_{j} x\right\| & \leqq \liminf _{n}\left\|P_{j}\left(x_{n}-x\right)\right\|,
\end{aligned}
$$

since $\operatorname{dim} X_{j}<\infty$ and $P_{j}$ is linear and continuous.

From (2.1) we have $\lim _{j} Q_{j} x=0$ and $\lim _{j} P_{j} x=x, x \neq 0$; then in (2.3)

$$
\begin{aligned}
& 0<c^{\prime} \leqq \liminf _{n}\left\|Q_{j}\left(x_{n}-x\right)\right\| \leqq \limsup _{n}\left\|Q_{j}\left(x_{n}-x\right)\right\| \leqq M, \\
& 0<c^{\prime \prime} \leqq \underset{n}{\liminf \left\|P_{j}\left(x_{n}-x\right)\right\|}
\end{aligned}
$$

holds for $j \lesssim j_{1}(x)$ with $c^{\prime}, c^{\prime \prime}$ and $M$ independent of $j$. Hence there exists $n_{0}(j)$ such that

$$
c^{\prime} / 2 \leqq\left\|Q_{j}\left(x_{n}-x\right)\right\| \leqq 2 M \text { and } 0<c^{\prime \prime} / 2 \leqq\left\|P_{j}\left(x_{n}-x\right)\right\|
$$

holds for $j \nsim j_{1}(x)$ and $n \geqq n_{0}(j)$. For the same $j$ and $n$,

$$
\left\|\frac{Q_{j}\left(x_{n}-x\right)}{\left\|Q_{j}\left(x_{n}-x\right)\right\|}\right\|=1 \text { and }\left\|\frac{P_{j}\left(x_{n}-x\right)}{\left\|Q_{j}\left(x_{n}-x\right)\right\|}\right\| \geqq c>0
$$

with $c$ independent of $j$ and $n$.

For $j \supset j_{0}, j \supset j_{1}(x), n \geqq n_{0}(j)$ and from (2.2) and (2.4) we deduce

$$
\left\|x_{n}-x\right\| \geqq(1+r)\left\|Q_{j}\left(x_{n}-x\right)\right\| .
$$

Hence from the first inequality in (2.3) and from (2.5)

$$
\liminf _{n}\left\|x_{n}-x\right\| \geqq(1+r)\left(1-\left\|Q_{j} x\right\|\right) .
$$

As we can choose $j$ large enough such that $(1+r)\left(1-\left\|Q_{j} x\right\|\right)>1$, we finally obtain $\lim \inf \left\|x_{n}-x\right\|>1$. Q.E.D.

All known examples of Banach spaces satisfying Opial's condition are isomorphic to uniformly convex Banach spaces; due to this the following two corollaries become interesting.

COROLlary 2.1. There exists a reflexive Banach space not isomorphic to any uniformly convex Banach space which satisfies Opial's condition. 
Proof. Let us put $X_{n}=R^{n}$ endowed with the norm $\left\|x_{n}\right\|_{n}=$ $\left(\sum_{i=1}^{n}\left|x_{n}(i)\right|^{n}\right)^{1 / n}, \quad x_{n}=\left(x_{n}(1), \cdots, x_{n}(n)\right) \in R^{n}$. The Hilbert product $X=\left\{x=\left(x_{n}\right)_{n=1,2, \ldots} ; x_{n} \in X_{n}\right.$ and $\left.\sum_{n=1}^{\infty}\left\|x_{n}\right\|_{n}^{2}<\infty\right\}$ is known to verify the first part of Corollary 2.1 (see [3]) for the norm $\|x\|=\left(\sum_{n=1}^{\infty}\left\|x_{n}\right\|_{n}^{2}\right)^{1 / 2}$. Condition (2.2) is easily proved for the projections

$$
\begin{aligned}
\left(P_{n} x\right)_{k} & =x_{k}, \quad k \leqq n, \\
& =0, \quad k>n,
\end{aligned}
$$

and $\left\{P_{n} ; n=1,2, \cdots\right\}$ forms an approximation of the identity. Q.E.D.

COROLlaRY 2.2. There exist nonreflexive Banach spaces which satisfy Opial's condition.

Proof. The usual Schauder basis of $l^{1}$ with its set of associated projections verify the hypothesis of Theorem 2.1. Q.E.D.

If we restrict a Banach space to be uniformly convex, the family can be asked to fulfill a more simple condition and we obtain the same conclusion.

THEOREM 2.2. Let $X$ be a uniformly convex Banach space with an approximation of the identity $\left\{P_{j} ; j \in J\right\}$ such that

$$
\lim _{j}\left\|I-P_{j}\right\|=1
$$

where $I$ is the identity on $X$. Then $X$ satisfies Opial's condition.

Proof. Let $x_{n} \rightarrow 0$ with $\lim \inf \left\|x_{n}\right\|=1$. From (2.7) we have for $x \in X$ and $Q_{j}=I-P_{j}$ that

$$
\left\|x_{n}-x\right\| \geqq\left\|Q_{j}\left(x_{n}-x\right)\right\|-\varepsilon \geqq\left\|x_{n}\right\|-\left(\left\|P_{j} x_{n}\right\|+\left\|Q_{j} x\right\|+\varepsilon\right),
$$

where $\varepsilon=\varepsilon(j)$ is independent of $n$ and $\lim _{j} \varepsilon(j)=0$. Then

$$
\underset{n}{\lim \inf }\left\|x_{n}-x\right\| \geqq 1-\left(\left\|Q_{j} x\right\|+\varepsilon\right),
$$

and taking limits on $j$ we obtain

$$
\underset{n}{\lim \inf }\left\|x_{n}-x\right\| \geqq 1
$$

for every $x$ in $X$. If for some $x \neq 0$ the equality holds in (2.8) then, $X$ being uniformly convex, we would have

$$
\underset{n}{\lim \inf }\left\|x_{n}-\frac{1}{2} x\right\|<1,
$$

contradicting (2.8), so the inequality is strict for $x \neq 0$ and from Lemma 1.1 we deduce the desired result. Q.E.D. 
A consequence of this theorem for $L^{p}$ spaces is the following

Corollary 2.3. Every Schauder basis $\left(e_{k}\right)_{k=1,2, \ldots}$ in $L^{p}(A)$, where $A$ is the unit real interval with Lebesgue measure and $1<p<\infty, p \neq 2$, is such that

$$
\underset{n}{\liminf }\left\|I-P_{n}\right\|>1
$$

for $P_{n}\left(\sum_{k=1}^{\infty} a_{k} e_{k}\right)=\sum_{k=1}^{n} a_{k} e_{k}$ and $I$ the identity.

Proof. $L^{p}(A)$ is uniformly convex and does not satisfy Opial's condition (see [9]), so (2.9) follows from Theorem 2.2. Q.E.D.

REMARK 1. The product $R \times l^{2}$ endowed with the norm $\|(a, y)\|=$ $\max (|a|,\|y\|)$ does not satisfy Opial's condition, even though $R$ and $l^{2}$ satisfy it. This can be shown by taking $x_{n}=\left(0, e_{n}\right)$, for $e_{n}$ the usual $n$th element of the basis of $l^{2}$. Then $\left\|x_{n}\right\|=1$ and $x_{n} \rightarrow 0$, but for $x=(1,0)$ we have $\left\|x_{n}-x\right\|=1$.

REMARK 2. Let us recall that a Banach space possesses normal structure if every convex and bounded subset $A$ of $X, A$ with diameter $\delta(A)>0$, has a point $x \in A$ such that $\sup _{y \in A}\|x-y\|<\delta(A)$. A similar condition to (2.2) was used in [4] for obtaining normal structure of a Banach space. Explicitly, for every $c>0$ there exists $r=r(c)>0$ such that

$$
\left\|P_{j} x\right\|=1 \text { and }\left\|x-P_{j} x\right\| \geqq c \Rightarrow\|x\| \geqq 1+r,
$$

where the family $\left\{P_{j}\right\}$ is an approximation of the identity. (2.10) does not imply Opial's condition as the example in Remark 1 shows it; however in [5] it is proved that Opial's condition implies normal structure.

3. Nonexpansive multivalued mappings. We apply Opial's condition to obtain a fixed point for a nonexpansive compact-valued mapping.

Definition 3.1. Let $C$ be a nonempty convex weakly compact subset of a Banach space $X$. A mapping $T: C \rightarrow K(X)$, where $K(X)$ denotes the family of nonempty compact subsets of $X$, is nonexpansive if

$$
D(T x, T y) \leqq\|x-y\|,
$$

for $x, y$ in $C$ and $D(\cdot, \cdot)$ the Hausdorff metrics on $K(X)$.

If we recall that the graph $G(U)$ of a multivalued mapping $U: A \rightarrow 2^{Y}$ is

$$
G(U)=\{(x, y) \in X \times Y ; x \in A, y \in U x\}
$$

we can prove the

THEOREM 3.1. Let $T: C \rightarrow K(X)$ be nonexpansive and let $X$ satisfy Opial's condition. Then the graph of $U=I-T$ is closed in $X, \sigma\left(X, X^{*}\right) \times(X,\|\cdot\|)$, where I denotes the identity on $X, \sigma\left(X, X^{*}\right)$ the weak topology and $\|\cdot\|$ the norm (or strong) topology. 
Proof. As the domain of $U$ is weakly compact we must prove that the graph is only sequentially closed. Let $\left(x_{n}, y_{n}\right) \in G(U)$ be such that

$$
x_{n} \rightarrow x, \quad y_{n} \rightarrow y .
$$

We must see that $x \in C$ and $y \in U x=x-T x$. That $x \in C$ is clear. As $y_{n} \in$ $x_{n}-T x_{n}$ we can write

$$
y_{n}=x_{n}-v_{n}, \quad v_{n} \in T x_{n} .
$$

From (3.1) we can find $v_{n}^{\prime} \in T x$ such that

$$
\left\|v_{n}-v_{n}^{\prime}\right\| \leqq\left\|x_{n}-x\right\| ;
$$

this is an easy consequence of the definition of Hausdorff metrics.

From (3.3) and (3.4) we obtain passing to limits on $n$

(3.5) $\quad \lim \inf \left\|x_{n}-x\right\| \geqq \lim \inf \left\|v_{n}-v_{n}^{\prime}\right\| \geqq \lim \inf \left\|x_{n}-y_{n}-v_{n}^{\prime}\right\|$.

$T x$ being compact and $y_{n} \rightarrow y$, for a convenient subsequence still denoted $v_{n}^{\prime}$ we have $v_{n}^{\prime} \rightarrow v \in T x$, so from (3.5)

$$
\lim \inf \left\|x_{n}-x\right\| \geqq \lim \inf \left\|x_{n}-y-v\right\| .
$$

As $x_{n} \rightarrow x$, Opial's condition implies that $y+v=x$, so $y=x-v \in x-T x=$ Ux. Q.E.D.

Let us recall the following known generalization of Picard's theorem:

Proposition 3.1 [7]. If $T: C \rightarrow K(C)$ is contractive, i.e. $D(T x, T y) \leqq$ $r\|x-y\|$ for $x, y$ in $C$ and $0<r<1$, then there exists a fixed point $x_{0} \in T x_{0}$.

We are now able to prove our fixed point theorem.

THEOREM 3.2. Let $X$ be a Banach space which satisfies Opial's condition. If $C$ is a nonempty convex weakly compact subset of $X$ and $T: C \rightarrow K(C)$ is a compact-valued nonexpansive mapping, then there exists a fixed point $x_{0} \in T x_{0}$.

Proof. Let $x^{\prime} \in C$ be fixed and define for $0<r_{m}<1$ and $r_{m} \rightarrow 1$

$$
T_{m} x=r_{m} T x+\left(1-r_{m}\right) x^{\prime}
$$

in an obvious way. Then $T_{m}: C \rightarrow K(C)$ and $T_{m}$ is contractive, so from Proposition 3.1 there exists a fixed point $x_{m} \in T_{m} x_{m}$. $C$ being weakly compact, for a convenient subsequence $\left\{x_{n}\right\}$ of $\left\{x_{m}\right\}$ we have $x_{n} \rightarrow x_{0} \in C$.

From (3.7) we deduce

so

$$
x_{n}=r_{n} v_{n}+\left(1-r_{n}\right) x^{\prime}, \quad v_{n} \in T x_{n}, x^{\prime} \in C,
$$

$$
\left\|x_{n}-v_{n}\right\|=\left(1-r_{n}\right)\left\|x^{\prime}-v_{n}\right\| .
$$


Then $y_{n}=x_{n}-v_{n} \in(I-T) x_{n}$ and $y_{n} \rightarrow 0$. If we put $U=I-T$, we have that $\left(x_{n}, y_{n}\right) \in G(U)$ and

$$
x_{n} \rightarrow x_{0}, \quad y_{n} \rightarrow 0 .
$$

From Theorem 3.1 we obtain

$$
0 \in U x_{0}=x_{0}-T x_{0},
$$

i.e. $x_{0} \in T x_{0}$ is a fixed point for $T$. Q.E.D.

REMARK. Recently Theorem 3.1 has been applied in [1] to obtain a generalization of Theorem 3.2 which only requires that $T$ be nonexpansive and sends the boundary of $C$ into compact subsets of $C$, this result can also be obtained from Theorem 3.1 and results on the topological degree for contractive multivalued mappings due to R. Nussbaum [8].

If we restrict the Banach space $X$ to be a Hilbert space we obtain a theorem due to J. Markin [7], and if we consider Banach spaces with a weakly continuous duality mapping we obtain a result of F. Browder [2], because these spaces satisfy Opial's condition [5].

\section{BIBLIOGRAPHY}

1. N. A. Assad and W. A. Kirk, Fixed point theorems for set-valued mappings of contractive type (to appear).

2. F. E. Browder, Nonlinear operators and nonlinear equations of evolution in Banach spaces, Proc. Sympos. Pure Math., vol. 18, part 2, Amer. Math. Soc., Providence, R.I. (to appear).

3. M. M. Day, Reflexive Banach spaces not isomorphic to uniformly convex spaces, Bull. Amer. Math. Soc. 47 (1941), 313-317. MR 2, 221.

4. J. P. Gossez et E. Lami Dozo, Structure normale et base de Schauder, Acad. Roy. Belg. Bull. Cl. Sci. (5) 55 (1969), 673-681. MR 41 \#795.

5. - Some geometric properties related to the fixed point theory for nonexpansive mappings, Pacific J. Math. 40 (1972), 565-573.

6. W. A. Kirk, A fixed point theorem for mappings which do not increase distances, Amer. Math. Monthly 72 (1965), 1004-1006. MR 32 \#6436.

7. J. T. Markin, A fixed point theorem for set valued mappings, Bull. Amer. Math. Soc. 74 (1968), 639-640. MR 37 \#3409.

8. R. Nussbaum, Some fixed point theorems, Bull. Amer. Math. Soc. 77 (1971), 360365.

9. Z. Opial, Weak convergence of the sequence of successive approximations for nonexpansive mappings, Bull. Amer. Math. Soc. 73 (1967), 591-597. MR 35 \#2183.

Department of Mathematics, University of Brussels, Brussels, Belgium 